\title{
Necessary and sufficient conditions for $R_{0}$ to be a sum of contributions of fertility loops
}

Claus Rueffler

Johan A.J. (Hans) Metz (metz@iiasa.ac.at)

\section{Approved by}

Ulf Dieckmann

Director, Evolution and Ecology Program

February 2015 Institute, its National Member Organizations, or other organizations supporting the work. 


\section{Necessary and sufficient conditions for $R_{0}$ to be a sum of contributions of fertility loops}

\section{Claus Rueffler · Johan A. J. Metz}

As a present to Odo Diekmann for his 65th birthday:

the answer to a question that he posed in a conversation sometime ago.

Abstract Recently, de-Camino-Beck and Lewis (2007, Bull Math Biol 69:13411354) have presented a method that under certain restricted conditions allows computing the basic reproduction ratio $R_{0}$ in a simple manner from life cycle graphs, without, however, giving an explicit indication of these conditions. In this paper, we give various sets of sufficient and generically necessary conditions. To this end, we develop a fully algebraic counterpart of their graphreduction method which we actually found more useful in concrete applications. Both methods, if they work, give a simple algebraic formula that can be interpreted as the sum of contributions of all fertility loops. This formula can be used in e.g. pest control and conservation biology, where it can complement sensitivity and elasticity analyses. The simplest of the necessary and sufficient conditions is that, for irreducible projection matrices, all paths from birth to reproduction have to pass through a common state. This state may be visible in the state representation for the chosen sampling time, but the passing may also occur in between sampling times, like a seed stage in the case of sampling just before flowering. Note that there may be more than one birth state, like when plants in their first year can already have different sizes at the sampling time. Also the common state may occur only later in life. However, in all cases $R_{0}$ allows a simple interpretation as the expected number of new individuals that in the next generation enter the common state deriving from a single in-

C. Rueffler

Mathematics and BioSciences Group, Department of Mathematics, University of Vienna, Nordbergstrasse 15, 1090 Vienna, Austria

E-mail: claus.rueffler@univie.ac.at

J. A. J. Metz

Mathematical Institute \& Institute of Biology, Leiden University, P.O. Box 9512, 2300RA Leiden, Netherlands

2nd affiliation: Netherlands Centre for Biodiversity, Naturalis, P.O. Box 9517, 2300RA Leiden, Netherlands

3rd affiliation: Evolution and Ecology Program, International Institute of Applied Systems Analysis, A-2361 Laxenburg, Austria E-mail: j.a.j.metz@biology.leidenuniv.nl dividual in this state. We end with pointing to some alternative algebraically simple quantities with properties similar to those of $R_{0}$ that may sometimes be used to good effect in cases where no simple formula for $R_{0}$ exists.

Keywords basic reproduction ratio, conservation, invasion, matrix models, population persistence, $R_{0}$

Mathematics Subject Classification (2000) MSC 92D15, MSC 92D25

\section{Introduction}

Matrix models are a popular tool in population dynamics (Caswell, 2001). Whenever individuals in a population can sensibly be grouped into a finite number of states such that transitions between these states occur at time intervals of fixed length, the dynamics of the population is described by

$$
\boldsymbol{N}(t+1)=\mathrm{A} \boldsymbol{N}(t),
$$

where $\mathrm{A}=\left[a_{l k}\right]$ is a nonnegative $n \times n$ population projection matrix and $\boldsymbol{N}(t)=\left(N_{1}(t), \ldots, N_{n}(t)\right)^{\mathrm{T}}$ is the column vector of densities in the different states at time $t$ (Caswell, 2001). The matrix entries $a_{l k}$ give the expected number of individuals in state $l$ at time $t+1$ that 'descend' from individuals in state $k$ at time $t$. This descent can occur either through the production of type- $l$ offspring by an individual in state $k$, denoted $f_{l k}$, or through the state-transition of an individual in state $k$ to state $l$, denoted $s_{l k}$, for example through growth or aging.

Of particular interest in many applications of matrix models, for instance in conservation ecology and pest control, is whether a given population can be expected to grow or shrink. This can be determined by computing the dominant eigenvalue $\lambda_{\mathrm{d}}$ of the population projection matrix A. A population, in the long run, will grow if $\lambda_{\mathrm{d}}>1$ and shrink if $\lambda_{\mathrm{d}}<1$.

An alternative method to evaluate population growth is to compute the basic reproduction ratio $R_{0}$ (Diekmann et al., 1990; Cushing and Yicang, 1994; Caswell, 2001; Li and Schneider, 2002). $R_{0}$ can be computed as the dominant eigenvalue of the next-generation matrix $\mathrm{G}=\left[g_{l k}\right]$, where the matrix entries $g_{l k}$ equal the expected number of offspring in birth state $l$ born over its life time to an individual with birth state $k$. Importantly, $R_{0}$ has the property

$$
R_{0} \lesseqgtr 1 \Longleftrightarrow \lambda_{\mathrm{d}} \lesseqgtr 1 \text {. }
$$

An advantage of using $R_{0}$ is that it often can be calculated analytically in situations where $\lambda_{d}$ can only be calculated numerically (e.g. Hurford et al 2010). In models with a single birth state, $R_{0}$ gives the number of offspring a newborn individual can expect over its life time.

Recently, de-Camino-Beck and Lewis $(2007,2008)$ presented an alternative method to calculate $R_{0}$. Their method is based on the reduction of the life cycle graph corresponding to $\mathrm{A}$. The advantage is that the resulting expression 
for $R_{0}$ has a simple and biologically useful interpretation: $R_{0}$ is given by a sum where each term describes the expected number of offspring obtained through a different reproductive pathway in the life cycle graph. de-Camino-Beck and Lewis argue that their formula can complement sensitivity and elasticity analysis in developing strategies for pest control and conservation. Unfortunately, $R_{0}$ can be written in this pleasing and useful form only if the next-generation matrix $G$ has a single non-zero eigenvalue. Only then additional insight can be expected from the application of the graph-reduction method. If $\mathrm{G}$ has two or more eigenvalues unequal zero, then $R_{0}$ can only be calculated explicitly by solving a polynomial of degree two or higher, resulting in an algebraically unpleasant expression that reveals little.

The purpose of this note is twofold. First, we derive a representation of the characteristic equation of the next generation matrix $G$ in terms of loops in the life cycle described by the population projection matrix $A$. If $G$ has only a single non-zero eigenvalue, then $R_{0}$ can immediately be written in a form where each term has the same useful biological interpretation as in the formula obtained with the graph-reduction method of de-Camino-Beck and Lewis $(2007,2008)$. Second, we derive various sets of sufficient and generically necessary conditions in terms of properties of loops in the life cycle such that $R_{0}$ is indeed the sole non-zero eigenvalue of the next-generation matrix $\mathrm{G}$. Only if these easily verifiable conditions are fulfilled it is worth to apply either the graph-reduction method of de-Camino-Beck and Lewis or the algebraic method presented here. Finally, we show that if $R_{0}$ can be expressed in a simple manner, then it also allows for a biologically intuitive interpretation that is independent of the number of birth states.

\section{Model and results}

Consider a population with $n$ discrete life history states, from now on called i-states (i for individual, cf. Metz and Diekmann 1986). Census is taken after time intervals of fixed length, just before reproduction. At reproduction individuals in i-state $k$ produce offspring of which on average $f_{l k} \geq 0$ are alive at the next census and in i-state $l$. After the reproduction episode all individuals - other than the newborns - undergo one of the following state-transitions: (i) with probability $s_{k k}$ they stay in their current i-state $k$, (ii) with probability $s_{l k}$ they move from i-state $k$ to i-state $l(l \neq k)$, (iii) with probability $d_{k}=1-\sum_{l} s_{l k}$ they die. Under these conditions, A can be decomposed into a fertility matrix $\mathrm{F}=\left[f_{l k}\right]$ and a state-transition matrix $\mathrm{S}=\left[s_{l k}\right]$ such that $\mathrm{A}=\mathrm{F}+\mathrm{S}$ (Caswell, 2001, p. 110). Normally, $f_{l k}$ and $s_{l k}$ are not constant but depend on the environment and change due to density dependence. Here, we restrict ourselves to constant matrices, i.e., focus on projection rather than prediction (Caswell, 2001, p. 30). The basic reproduction ratio is now defined as

$$
R_{0}=\rho\left(\mathrm{F}(\mathrm{I}-\mathrm{S})^{-1}\right)
$$

(Cushing and Yicang, 1994; Caswell, 2001; Diekmann et al., 1990; Li and Schneider, 2002). Here $\rho$ denotes the spectral radius (the largest modulus of all eigenvalues), I the identity matrix and the exponent -1 matrix inversion. The matrix $\mathrm{G}=\left[g_{l k}\right]=\mathrm{F}(\mathrm{I}-\mathrm{S})^{-1}$ is sometimes called fundamental matrix (Caswell, 2001) or next-generation matrix (Diekmann et al., 1990; Li and Schneider, 2002). Each entry $g_{l k}$ gives the expected number of offspring in birth state $l$ that are born over its life time to an individual that was itself born in birth state $k$. Thus, the matrix $\mathrm{G}$ has as many rows with non-zero entries as there are birth states. As a consequence, the number of birth states is an upper limit for the number of non-zero eigenvalues of $\mathrm{G}$. This is one reason why $R_{0}$ can often be calculated analytically in situations where $\lambda_{\mathrm{d}}$ can only be calculated numerically.

What is the interpretation of $R_{0}$ ? In age-structured populations all newborns enter the same birth state and $R_{0}$ gives the number of offspring a newborn individual can expect over its life time. This interpretation holds in all life cycles with only a single birth state. In life cycles with more than one birth state, as those shown in Fig. 1 and 2, this interpretation can be generalized. Then $R_{0}$ is the expected number of offspring of a newborn individual where the expectation has also to be taken over the possible birth states of the newborn individual whose offspring we are counting (Diekmann et al., 1990). This interpretation follows directly from the eigenvalue equation. Let $\boldsymbol{u}$ denote the normalized right eigenvector corresponding to $R_{0}$ (where $\boldsymbol{u}$ is a column vector). The $l$ th entry of $\boldsymbol{u}$ gives the frequency of individuals born in i-state $l$ once the population has reached its stable i-state distribution. Then, by definition, $\mathbf{G} \boldsymbol{u}=R_{0} \boldsymbol{u}$. The entries in the vector $\mathrm{G} \boldsymbol{u}$ give the expected number of offspring in the different birth states of a 'distributed' individual represented by $\boldsymbol{u}$. Multiplying both sides with $\mathbf{1}^{\mathrm{T}}$ (the row vector consisting of only $1^{\prime} \mathrm{s}$ ) to get the expected number of offspring of a distributed individual gives $\mathbf{1}^{\mathrm{T}} \mathrm{G} \boldsymbol{u}=R_{0}$.

2.1 A new tool for calculating $R_{0}$

Generally, $R_{0}$ can be computed from Eq. (3), either analytically, if there are few birth states, or numerically. Here we derive, under restrictive conditions to be detailed further in Subsections 2.2 and 2.3, a formula for $R_{0}$ such that the resulting expression is a sum where each term describes the contribution from a single fertility loop. Before proceeding we introduce some terminology with respect to life cycle graphs. A loop is a sequence of demographic parameters $s_{l k}, f_{l k}$ that lead from an i-state in the life cycle to itself without passing through any i-state more than once. The loop transmission $L$ equals the product of the demographic parameters along the loop. In the remainder of this paper we will use the terms loop and loop transmission synonymously and often denote a loop with its transmission. The set of all loops in a specific life cycle corresponding to a population projection matrix $A$ is denoted by $\mathcal{L}_{\mathrm{A}}$. As an example consider the life cycle shown in Fig. 2(a) where $\mathcal{L}_{\mathrm{A}}=\left\{f_{13} s_{31}, f_{24} s_{42}, f_{13} s_{32} f_{24} s_{41}\right\}$. A fertility loop is a loop that contains at 
least one fertility parameter $f_{l k}$ (in the above set all loops are fertility loops). Fertility loops are collected in the set $\mathcal{L}_{\mathrm{f}, \mathrm{A}}$. A state-transition loop is a loop that only contains transition probabilities $s_{l k}$ and no fertility parameter. These are collected in the set $\mathcal{L}_{\mathrm{S}}$. By $\breve{L}$ we denote the set of all i-states that are traversed by loop $L$ (e.g. if $L=f_{13} s_{31}$, then $\breve{L}=\{1,3\}$ ) and by $|L|_{\mathrm{f}}$ the number of fertility parameters $f_{l k}$ occurring in loop $L$ (e.g. if $L=f_{13} s_{32} f_{24} s_{41}$, then $\left.|L|_{\mathrm{f}}=2\right)$. Two loops $L$ and $M$ are connected if they share at least one i-state, i.e., $\breve{L} \cap \breve{M} \neq \emptyset$ (the loops $f_{13} s_{31}$ and $f_{13} s_{32} f_{24} s_{41}$ are connected because they both pass through the first and third i-state) and unconnected otherwise (such as $f_{13} s_{31}$ and $f_{24} s_{42}$ ). With this notation we can write the characteristic equation of $\mathrm{G}$ as a combination of loops $L, M, N \ldots$ in the following way:

$$
\begin{aligned}
1=\left(\sum_{\mathcal{L}_{\mathrm{f}, \mathrm{A}}} L z^{-|L|_{\mathrm{f}}}-\sum_{\mathcal{L}_{\mathrm{f}, \mathrm{A}} \times \mathcal{L}_{\mathrm{A}}} L M z^{-\left(|L|_{\mathrm{f}}+|M|_{\mathrm{f}}\right)}\right. \\
\left.\quad+\sum_{\mathcal{L}_{\mathrm{f}, \mathrm{A}} \stackrel{*}{\times} \mathcal{L}_{\mathrm{A}}{ }^{*} \times \mathcal{L}_{\mathrm{A}}} L M N z^{-\left(|L|_{\mathrm{f}}+|M|_{\mathrm{f}}+|N|_{\mathrm{f}}\right)}-\ldots\right) / \operatorname{det}(\mathrm{I}-\mathrm{S})
\end{aligned}
$$

(Appendix A). Each sum runs over the $k$-fold Cartesian product of $\mathcal{L}_{\mathrm{f}, \mathrm{A}}$ with $k-1$ times $\mathcal{L}_{\mathrm{A}}$, where $k$ equals one in the first summation and increases by one with each subsequent summation. An asterisk indicates that only pairs, triplets, etc. of mutually unconnected loops are considered in the Cartesian product. Thus, $k$ only increases up to the highest number of mutually unconnected loops existing in the life cycle. Each loop $L$ is multiplied with the scalar $z^{-j}$ where $j=|L|_{\mathrm{f}}$

By definition of the characteristic equation, the numbers $z$ for which Eq. (4) holds true are the eigenvalues of the next-generation matrix $G$ and the eigenvalue with the largest modulus equals $R_{0}$. If $n$ is the number of i-states in a life cycle (and therefore the dimension of the matrices $A$ and $G$ ), then number $m \leq n$ exists such that the matrix $\mathrm{G}$ has $m$ eigenvalues unequal to zero and $n-m$ eigenvalues equal to zero. If $m \geq 2$ calculating an explicit expression for $R_{0}$ requires determining the roots of a polynomial of degree 2 or higher, resulting in an algebraically complex and biologically unrevealing formula for $R_{0}$. If, however, $m=1$, then $\mathrm{G}$ has only a single non-zero eigenvalue, which we immediately identify as $R_{0}$. In this case, by multiplying both sides of Eq (4) with $z$ and replacing $z$ with $R_{0}$, we obtain

$$
R_{0}=\left(\sum_{\mathcal{L}_{\mathrm{f}, \mathrm{A}}} L-\sum_{\mathcal{L}_{\mathrm{f}, \mathrm{A}} \times \mathcal{L}_{\mathrm{S}}} L M+\sum_{\mathcal{L}_{\mathrm{f}, \mathrm{A}} \stackrel{*}{\times} \mathcal{L}_{\mathrm{S}} \times \mathcal{L}_{\mathrm{S}}^{*}} L M N-\ldots\right) / \operatorname{det}(\mathrm{I}-\mathrm{S}),
$$

which can be simplified to (Appendix A)

$$
R_{0}=\sum_{\mathcal{L}_{\mathrm{f}, \mathrm{A}}} L \frac{\operatorname{det}\left(\mathrm{I}-\mathrm{S}_{\backslash \breve{L}}\right)}{\operatorname{det}(\mathrm{I}-\mathrm{S})} .
$$

Here, $\mathrm{S}_{\breve{L}}$ denotes the sub-matrix obtained by deleting all rows and columns in the state-transition matrix $\mathrm{S}$ that correspond to i-states passed by the loop $L$. Eq. (6) constitutes one of our main results: $R_{0}$ is given as a weighted sum of fertility loops. This expression is similar to the one obtained by the graphreduction method of de-Camino-Beck and Lewis (2007, 2008).

Under certain conditions the weighting factors can be simplified further. Before presenting these simplifications we review some matrix algebra. A nonnegative matrix is called reducible if there exists a pair of i-states such that no path exists leading from one i-state to the other. For example, the statetransition matrix $\mathrm{S}$ corresponding to the life cycle shown in Fig. 1 is reducible because when starting in i-state 5 the i-states 1,2 or 3 can only be reached through the fertility parameter $f_{15}$. By renumbering the i-states in the life cycle any $n$-dimensional reducible state-transition matrix $\mathrm{S}$ can be brought into the form of a block triangular matrix such that the matrices on the diagonal of the block triangular matrix are irreducible (=not reducible) (Horn and Johnson, 1985; Caswell, 2001). We denote the square matrices on the diagonal of this block triangular matrix by $\mathrm{S}_{n^{i}}$. Thus, the $n$ i-states $\{1, \ldots, n\}$ of a life cycle can be grouped into $m$ subsets $\boldsymbol{n}^{1}=\left\{n_{1}^{1}, n_{2}^{1} \ldots\right\}, \ldots, \boldsymbol{n}^{m}=\left\{n_{1}^{m}, n_{2}^{m} \ldots\right\}$ such that the matrices $S_{\boldsymbol{n}^{i}}$ are irreducible. Then $\operatorname{det}(\mathbf{I}-\mathrm{S})=\prod_{i=1}^{m} \operatorname{det}\left(\mathbf{I}-\mathrm{S}_{\boldsymbol{n}^{i}}\right)$ (Horn and Johnson, 1985). Thus, we can rewrite Eq. (6) as

$$
R_{0}=\sum_{\mathcal{L}_{\mathrm{f}}} L \frac{\prod_{\mathcal{D}_{\mathrm{s}}} \operatorname{det}(\mathrm{I}-\mathrm{D})}{\prod_{\mathcal{D}_{\mathrm{S}}} \operatorname{det}(\mathrm{I}-\mathrm{D})},
$$

where $\mathcal{D}_{\mathrm{S}}$ and $\mathcal{D}_{\mathrm{S}_{\breve{L}}}$ denote the sets of irreducible matrices on the diagonal of the block triangular matrices corresponding to $S$ and $S_{\backslash \breve{L}}$, respectively. Note that for any zero matrix $D$ we have $\operatorname{det}(I-D)=1$ and so we can forget about them. If $\mathcal{D}_{\mathrm{S}} \cap \mathcal{D}_{\mathrm{S}_{\breve{L}}} \neq \emptyset$, then there exist factors $\operatorname{det}(\mathrm{I}-\mathrm{D})$ that occur in both the numerator and denominator of the right-hand side of Eq. (7) and thus cancel out. The final formula for $R_{0}$ becomes

$$
R_{0}=\sum_{\mathcal{L}_{\mathrm{f}}} L \frac{\prod_{\mathcal{D}_{\mathrm{S}_{\backslash \breve{L}} \backslash \mathcal{D}_{\mathrm{S}}}} \operatorname{det}(\mathrm{I}-\mathrm{D})}{\prod_{\mathcal{D}_{\mathrm{S}} \backslash \mathcal{D}_{\mathrm{S}_{\backslash} \backslash \breve{L}}} \operatorname{det}(\mathrm{I}-\mathrm{D})} .
$$

However, when it comes to calculating $R_{0}$ for concrete models it is usually easier to compute $R_{0}$ according to Eq. (6) and then do simplifications of the weighting terms $\operatorname{det}\left(I-S_{\breve{L}}\right) / \operatorname{det}(I-S)$ "by hand" instead of going through the algebraic procedure leading to Eq. (8). 
2.2 When does the tool work and how to interpret the result

Under which conditions does the next-generation matrix $\mathrm{G}$ have a single nonzero eigenvalue, or, asked differently, under which conditions can we hope to gain additional biological insights from applying either the graph-reduction method of de-Camino-Beck and Lewis $(2007,2008)$ or its algebraic counterpart presented here? As discussed above, $G$ has a single non-zero eigenvalue if the numerator on the right-hand side of Eq. (4) is linear in $z^{-1}$. In terms of properties of loops we can state this condition as follows.

\section{Theorem 1. If}

(i) all fertility loops contain exactly one fertility parameter and

(ii) all fertility loops are mutually connected,

then the next-generation matrix $\mathrm{G}$ has a single non-zero eigenvalue.

These conditions are easy to check for any life cycle and we leave it to the reader to verify that the example shown in Fig. 1 fulfills both conditions while the example shown in Fig. 2(a) violates both conditions. Thus, under the conditions of Theorem 1, $R_{0}$ can be calculated according to Eq. (6). Importantly, these conditions do not imply the existence of only a single birth state. And indeed, the life cycle in Fig. 1 has two birth states. Thus, maybe somewhat surprisingly, the next-generation matrix $G$ can have a single non-zero eigenvalue also in models with multiple birth states.

The conditions in Theorem 1 can be formulated in a different but equivalent manner.

Lemma 2. If all fertility loops contain only a single fertility parameter, then all fertility loops are mutually connected if and only if an i-state exists that is passed by all fertility loop

Proof. See Appendix B.

This allows us to reformulate Theorem 1 as follows.

Theorem 3. If

(i) all fertility loops contain exactly one fertility parameter and

(ii) an $i$-state exists that is passed by all fertility loops,

then the next-generation matrix $\mathrm{G}$ has a single non-zero eigenvalue.

An example for a life cycle with multiple birth states in which all fertility loops pass through a common i-state is shown in Fig. 1. Here, all individuals pass the i-state of small adults. For the case of irreducible population projection matrices A we can reformulate Condition (i) and (ii) from Theorem 3 in an even more pleasing manner.

Theorem 4. If A is irreducible, then all fertility loops contain only a single fertility parameter and are mutually connected, if and only if there exist an i-state through which all newborns have to pass before they can reproduce.

Proof. See Appendix C.
The existence of an i-state that is passed by all fertility loops allows for a simple and biologically intuitive interpretation for $R_{0}$ that is independent of the number of birth states.

Proposition 5. Under the conditions of Theorem 3 the single non-zero eigenvalue of $\mathrm{G}, R_{0}$, gives the expected number of new individuals that in the next generation enter the common state deriving from a single individual in this i-state.

Proof. See Appendix D.

Remark. One may interpret this result by introducing generalized individuals (Metz, submitted) defined in the following manner. A generalized individual is born at the moment an ordinary individual enters the common i-state. It is then composed of this ordinary individual and all of its offspring till the latter enter the common i-state. With the introduction of these generalized individuals we are back in the comfortable situation of having a single birth state so that $R_{0}$ can be interpreted as the expected number of offspring without having to average over birth states.

Theorem 3 is formulated as a unidirectional implication. Thus, the conditions given in the Theorem are sufficient. However, they are not necessary because it is in principle possible that for each $j>1$ all summands on the right-hand side of Eq. (4) containing the factor $z^{-j}$ cancel out so that only terms containing $z^{-1}$ remain. To analyze this possibility systematically we subdivide the set $\mathcal{A}$ of all possible population projection matrices $\mathrm{A}$ in the following way. An $n \times n$ population projection matrix A can be decomposed into a state-transition matrix $\mathrm{S}$ and a fertility matrix $\mathrm{F}$. These matrices are characterized by the distribution of zero and non-zero entries, that is, by the possible transitions $s_{l k}, f_{l k}>0$. Furthermore, $\mathrm{S}$ and $\mathrm{F}$ are characterized by the exact numerical values of the non-zero entries. Let $Y_{1}=\left\{(k, l): s_{l k}=0\right\}$ and $Y_{2}=\left\{(k, l): f_{l k}=0\right\}$ denote the set of positions in $S$ and $F$, respectively where these matrices contain zeros. The number of non-zero entries in $\mathrm{S}$ and $\mathrm{F}$ is given by $n_{1}=n \times n-\left|Y_{1}\right|$ and $n_{2}=n \times n-\left|Y_{2}\right|$, respectively. Then a triple $\boldsymbol{y}=\left(n, Y_{1}, Y_{2}\right) \in \boldsymbol{Y}:=\bigcup_{n=1}^{\infty}\{n\} \times \mathcal{P}\left(\{1, \cdots, n\}^{2}\right) \times \mathcal{P}\left(\{1, \cdots, n\}^{2}\right)$, with $\mathcal{P}$ denoting the power set, determines the dimension of the matrices $\mathrm{S}$ and $\mathrm{F}$ and the positions where these matrices contain zeros. Let $\mathcal{A}_{\boldsymbol{y}} \subset \mathcal{A}$ denote the set of all matrices that fulfill the constraints specified by $\boldsymbol{y}$. A particular realization of $\mathrm{A} \in \mathcal{A}_{\boldsymbol{y}}$ can be described by a vector $\boldsymbol{x} \in \boldsymbol{X}_{\boldsymbol{y}}:=(0,1]^{n_{1}} \times \mathbb{R}_{>0}^{n_{2}}$ specifying the non-zero matrix entries of $S$ and $F$. With this notation we can describe the set $\mathcal{A}$ of all possible population projection matrices $\mathrm{A}$ as a nested family of models. The outer family is given by

$$
\mathcal{A}=\left\{\mathcal{A}_{\boldsymbol{y}}: \boldsymbol{y} \in \boldsymbol{Y}\right\}
$$

in which each element defines an inner family

$$
\mathcal{A}_{\boldsymbol{y}}=\left\{\mathrm{A}: \boldsymbol{x} \in \boldsymbol{X}_{\boldsymbol{y}}\right\}
$$


Consider a constraint $\boldsymbol{y} \in \boldsymbol{Y}$. Then, generically in $\boldsymbol{X}_{\boldsymbol{y}}$, i.e. for all $\boldsymbol{x} \in \boldsymbol{X}_{\boldsymbol{y}}$ except for a nowhere dense (as well as Lebesgue measure zero) subset, not all summands containing $z^{-j}, j>1$, in the numerator on the right-hand side of Eq. (4) cancel out. Thus, we can formulate a stronger version of Theorem 3 and Theorem 4 .

Theorem 6. The next generation matrix $\mathrm{G}$ has a single non-zero eigenvalue if and generically in $\boldsymbol{X}_{\boldsymbol{y}}$ only if $\boldsymbol{y}$ is such that

(i) all fertility loops contain exactly one fertility parameter and

(ii) an i-state exists that is passed by all fertility loops.

Theorem 7. If A is irreducible, then the next generation matrix $\mathrm{G}$ has a single non-zero eigenvalue if and generically in $\boldsymbol{X}_{\boldsymbol{y}}$ only if $\boldsymbol{y}$ is such that there exis an $i$-state through which all newborns have to pass before they can reproduce.

\subsubsection{Hidden i-states}

In the following we take a closer look at the genericity argument used in Theorem 6 and Theorem 7. It appears that a cancellation of terms can occur under certain rank constraints on the matrices $S$ and F. For instance, from Eq. (3) it is clear that $\operatorname{rank} F=1$ implies $\operatorname{rank} G=1$ which in turn implies that $G$ has a single non-zero eigenvalue. The rank of $F$ depends on the numerical values of the entries $f_{l k}$ and $\operatorname{rank} F=1$ can be fulfilled or not fulfilled for the same life cycle and irrespective of the conditions in Theorem 3.

Although for any $n \times n$ population projection matrix $\mathrm{A}$ with $n>1$ we have $\operatorname{rank} \mathrm{G}=1$ if and only if $\operatorname{rank} \mathrm{F}=1\left(\operatorname{since} \operatorname{rank}(\mathrm{I}-\mathrm{S})^{-1}=n\right)$ a linear characteristic equation can also exist when $\operatorname{rank} F>1$. For this it is necessary that $G$ does not have a complete basis of eigenvectors. Such matrices are known as defective (Horn and Johnson, 1985). It is then possible that the algebraic multiplicity of the eigenvalue zero equals $n-1$ while its geometric multiplicity equals $n-\operatorname{rank} \mathrm{F}$. For instance, let A be an $n \times n$ population projection matrix corresponding to a life cycle in which individuals in a birth state cannot reproduce. In particular, $f_{k k}=0$ for all $k \in\{1, \ldots, n\}$. Furthermore, assume that sexually mature individuals cannot enter a birth state through a state-transition. If we number the i-states such that all birth states have indices in $\{1, \ldots, m\}$ while i-states that are able to reproduce have indices in $\{m+1, \ldots, n\}$, this means that $s_{l k}=0$ for all $l \in\{1, \ldots, m\}$ and $k \in\{m+1, \ldots, n\}$. Then $\mathrm{A}$ can be written as

$$
A=S+F=\left(\begin{array}{cc}
S_{11} & 0 \\
S_{21} & S_{22}
\end{array}\right)+\left(\begin{array}{cc}
0 & F_{12} \\
0 & 0
\end{array}\right)
$$

where $\mathrm{S}_{11}$ and $\mathrm{S}_{22}$ are $m \times m$ - and $n-m \times n-m$-matrices, respectively. Let us assume rank $S_{21}=1$. This is the case if and only if two vectors $\boldsymbol{u}_{1}$ and $\boldsymbol{u}_{2}$ exist such that $\boldsymbol{u}_{2} \boldsymbol{u}_{1}^{\mathrm{T}}=\mathrm{S}_{21}$. In this model, $\mathrm{G}$ has a single non-zero eigenvalue regardless of rank $F_{12}$ (Appendix $\mathrm{E}$ ). This eigenvalue equals

$$
R_{0}=\boldsymbol{u}_{1}^{\mathrm{T}}\left(\mathrm{I}-\mathrm{S}_{11}\right)^{-1} \mathrm{~F}_{12}\left(\mathrm{I}-\mathrm{S}_{22}\right)^{-1} \boldsymbol{u}_{2} .
$$

Rank constraints as discussed above can be fulfilled for two different reasons. A matrix of rank 1 can occur because the matrix entries just happened to be fine-tuned in a very specific manner. Since there is no reason to expect such fine-tuned parameters in nature this case is of no biological interest. It is these cases that we want to exclude with the genericity clause. However, a matrix of rank 1 can also occur as a consequence of a hidden i-state that is passed by all individuals that make the transitions described by the matrix under consideration. With hidden i-states we mean states that are not present at the sampling time but at some time point between two sampling steps. For instance, assume that a hidden i-state $H$ exists that is passed at some time point $t+\Delta$ between two censuses at time $t$ and $t+1$ by all individuals that make a transition from i-states in $\beta=\left\{\beta_{1}, \beta_{2}, \ldots\right\} \subseteq\{1, \ldots, n\}$ to i-states in $\alpha=\left\{\alpha_{1}, \alpha_{2}, \ldots\right\} \subseteq\{1, \ldots, n\}$. Then two vectors $\boldsymbol{s}_{1}^{\mathrm{T}}=\left(s_{\alpha_{1} H}, s_{\alpha_{2} H}, \ldots\right)$ and $\boldsymbol{s}_{2}^{\mathrm{T}}=\left(s_{H \beta_{1}}, s_{H \beta_{2}}, \ldots\right)$ exist such that transitions from i-states in $\beta$ to i-states in $\alpha$ are described by the matrix $\mathrm{S}_{\alpha, \beta}:=s_{1} s_{2}^{\mathrm{T}}$ with $\operatorname{rank} \mathrm{S}_{\alpha, \beta}=1$.

Hidden i-states can be incorporated in our loop formulation. Consider an $n \times n$ population projection matrix $\mathrm{A}$. Assume that at time $t+\Delta$ the state space has dimension $m$. Thus, an $m \times n$ population projection matrix $\mathrm{A}^{\prime}$ exists that projects the population from time $t$ to time $t+\Delta$ and an $n \times m$ population projection matrix $\mathrm{A}^{\prime \prime}$ exists that projects the population from time $t+\Delta$ to time $t+1$. Then

$$
\boldsymbol{N}(t+1)=\mathrm{A}^{\prime \prime} \mathrm{A}^{\prime} \boldsymbol{N}(t)
$$

The life cycle with the hidden i-states made explicit is given by the matrix

$$
B=\left(\begin{array}{cc}
0 & A^{\prime \prime} \\
A^{\prime} & 0
\end{array}\right)
$$

Taking hidden i-states into account can have consequences for which sequences of demographic parameters actually qualify as loops. For instance, if all newborns pass through the same hidden i-state $H$ independent of the i-state of their parents at time $t$ and independent of their own i-state at time $t+1$, then $f_{l k}=s_{l H} f_{H k}$ and $f_{k l}=s_{k H} f_{H l}$, and a loop $f_{l k} f_{k l}$ in the life cycle corresponding to A becomes a non-loop in the life cycle corresponding to $\mathrm{B}$. Thus, the life cycle corresponding to B can contain less loops than the life cycle corresponding to A. Similarly, under this condition any two unconnected fertility loops in the life cycle corresponding to $\mathrm{A}$ like $f_{11}$ and $f_{22}$ become connected in the life cycle corresponding to $\mathrm{B}$. Crucially, the value for $R_{0}$ does not change when hidden i-states are incorporated into the loop formulation.

The characteristic equation for the next generation matrix $G$ as derived from matrix $\mathrm{B}$ equals Eq. (4) where $\mathcal{L}_{\mathrm{A}}$ and $\mathcal{L}_{\mathrm{f}, \mathrm{A}}$ have to be replaced with $\mathcal{L}_{\mathrm{B}}$ and $\mathcal{L}_{\mathrm{f}, \mathrm{B}}$, respectively. In particular, if a time interval $\Delta$ and an i-state $H$ that is visible at time $t+\Delta$ exist such that all fertility loops $L \in \mathcal{L}_{\mathrm{f}, \mathrm{B}}$ contain only a single fertility parameter and pass through $H$, then $R_{0}$ is given by Eq. $(6)$ with $\mathcal{L}_{\mathrm{f}, \mathrm{A}}$ replaced by $\mathcal{L}_{\mathrm{f}, \mathrm{B}}$. Thus, Theorem 6 and Theorem 7 can be reformulated in terms of $B$ instead of $A$. In this manner, we could given sufficient and generically necessary conditions for $\mathrm{G}$ to have a single non-zero eigenvalue that takes the existence of hidden i-states explicitly into account. 
In the following, we express Theorem 6 and Theorem 7 in terms of matrices A while simultaneously taking account of rank constraints resulting from hidden i-states. To this end, we extend the classification of population projection matrices A given by Eq. (9) and Eq. (10). Consider an organism with a life cycle specified by the constraints given in $\boldsymbol{y} \in \boldsymbol{Y}$. Let $\boldsymbol{H}$ denote the set of hidden i-states in the life cycle of the organism under consideration that impose rank1-constraints on sub-matrices of $\mathrm{S}$ or $\mathrm{F}$. The set of all matrices $\mathrm{A} \in \mathcal{A}_{\boldsymbol{y}}$ that fulfill the rank-constraints imposed by $\boldsymbol{H}$ is denoted $\mathcal{A}_{\boldsymbol{y}, \boldsymbol{H}}$. A particular realization of $\mathrm{A} \in \mathcal{A}_{\boldsymbol{y}, \boldsymbol{H}}$ can be described by a vector $\boldsymbol{x} \in \boldsymbol{X}_{\boldsymbol{y}, \boldsymbol{H}} \subset(0,1]^{n_{1}} \times \mathbb{R}_{>0}^{n_{2}}$ specifying the non-zero entries of $\mathrm{S}$ and $\mathrm{F}$. With this notation we can state the following results.

Theorem 8. The next generation matrix $\mathrm{G}$ has a single non-zero eigenvalue if and generically in $\boldsymbol{X}_{\boldsymbol{y} \cdot \boldsymbol{H}}$ only if $\boldsymbol{y}$ and $\boldsymbol{H}$ are such that

(i) all fertility loops $L \in \mathcal{L}_{\mathrm{f}, \mathrm{A}}$ contain exactly one fertility parameter and

(ii) an i-state exists, overt or hidden, that is passed by all fertility loops.

Theorem 9. If $\mathrm{A}$ is irreducible, then the next generation matrix $\mathrm{G}$ has a single non-zero eigenvalue if and generically in $\boldsymbol{X}_{\boldsymbol{y}, \boldsymbol{H}}$ only if $\boldsymbol{y}$ and $\boldsymbol{H}$ are such that there exist an $i$-state, overt or hidden, through which all newborns have to pass before they can reproduce.

Proposition 10. Under the conditions of Theorem 8 the single non-zero eigenvalue of $\mathrm{G}, R_{0}$, gives the expected number of new individuals that in the next generation enter the overt or hidden common i-state deriving from a single individual in this $i$-state.

We conclude with a remark on the calculation of $R_{0}$. If a hidden i-state is known that is passed by all fertility loops, then it is not necessary to construct the full matrix B and derive Eq. (5) from it. Instead, it suffices to add the hidden i-state to the life cycle given by $A$ and then calculate Eq. (5). This procedure is applied in the second example in the following section.

\section{Two examples}

In this section we apply the formalism presented here to two examples. In the first example, we consider the life cycle of creeping avens (Geum reptans) as described by Weppler et al. (2006). This life cycle is shown in Fig. 1. It consists of the following fertility loops: $L_{1}=f_{33}, L_{2}=f_{34} s_{43}, L_{3}=f_{35} s_{54} s_{43}, L_{4}=$ $f_{13} s_{32} s_{21}, L_{5}=f_{14} s_{43} s_{32} s_{21}$ and $L_{6}=f_{15} s_{54} s_{43} s_{32} s_{21}$. The state-transition matrix $\mathrm{S}$ equals

$$
\mathbf{S}=\left(\begin{array}{ccccc}
0 & 0 & 0 & 0 & 0 \\
s_{21} & s_{22} & 0 & 0 & 0 \\
0 & s_{32} & 0 & 0 & 0 \\
0 & 0 & s_{43} & s_{44} & s_{45} \\
0 & 0 & 0 & s_{54} & s_{55}
\end{array}\right)
$$

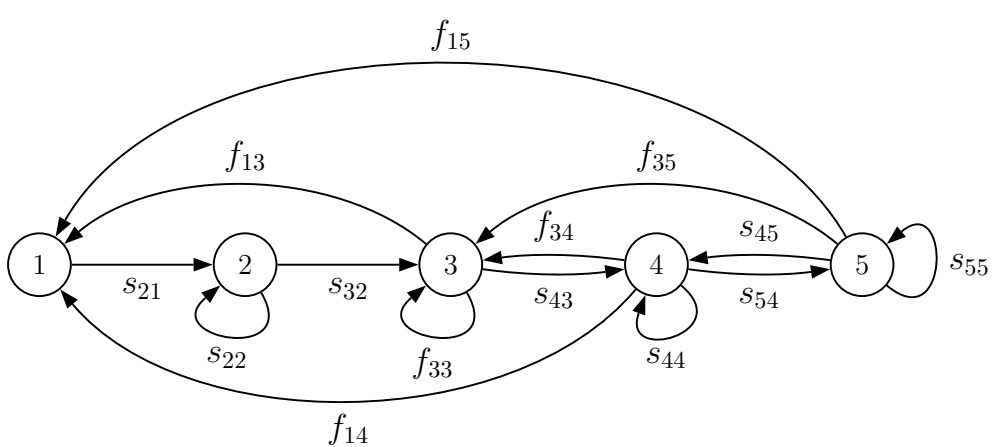
Fig. 1 Life cycle graph of creeping avens (Geum reptans) as described by Weppler et al.
$(2006)$ with seedling (1), juvenile (2), small adult (3), medium adult (4) and large adult i-state (5).

from which follows immediately that

$$
\begin{aligned}
\operatorname{det}(\mathrm{I}-\mathrm{S}) & =\left(1-s_{22}\right)\left(\left(1-s_{44}\right)\left(1-s_{55}\right)-s_{45} s_{54}\right) \\
\operatorname{det}\left(\mathrm{I}-\mathrm{S}_{\backslash \breve{L}_{1}}\right) & =\left(1-s_{22}\right)\left(\left(1-s_{44}\right)\left(1-s_{55}\right)-s_{45} s_{54}\right) \\
\operatorname{det}\left(\mathrm{I}-\mathrm{S}_{\backslash \breve{L}_{2}}\right) & =\left(1-s_{22}\right)\left(1-s_{55}\right) \\
\operatorname{det}\left(\mathrm{I}-\mathrm{S}_{\backslash \breve{L}_{3}}\right) & =\left(1-s_{22}\right) \\
\operatorname{det}\left(\mathrm{I}-\mathrm{S}_{\backslash \breve{L}_{4}}\right) & =\left(1-s_{44}\right)\left(1-s_{55}\right)-s_{45} s_{54} \\
\operatorname{det}\left(\mathrm{I}-\mathrm{S}_{\backslash \breve{L}_{5}}\right) & =\left(1-s_{55}\right) \\
\operatorname{det}\left(\mathrm{I}-\mathrm{S}_{\backslash \breve{L}_{6}}\right) & =1 .
\end{aligned}
$$

Thus, Eq. (6) becomes

$$
\begin{aligned}
R_{0}= & f_{33}+\frac{f_{34} s_{43}}{\left(1-s_{44}\right)-s_{45} s_{54} /\left(1-s_{55}\right)}+\frac{f_{35} s_{54} s_{43}}{\left(1-s_{44}\right)\left(1-s_{55}\right)-s_{45} s_{54}} \\
& +\frac{f_{13} s_{32} s_{21}}{1-s_{22}}+\frac{f_{43} s_{32} s_{21}}{\left(1-s_{22}\right)\left(\left(1-s_{44}\right)-s_{45} s_{54} /\left(1-s_{55}\right)\right)} \\
& +\frac{f_{15} s_{54} s_{43} s_{32} s_{21}}{\left(1-s_{22}\right)\left(\left(1-s_{44}\right)\left(1-s_{55}\right)-s_{45} s_{54}\right)}
\end{aligned}
$$

In this equation, each summand gives the expected number of offspring due to a specific fertility loop. Note that newborns either appear as seedlings (istate 1 ) or as small adults (i-state 3 ). Note furthermore that all fertility loops pass the i-state of small adults. Thus, $R_{0}$ as described by Eq. (13) gives the expected number of small adults a single small adult is expected to produce.

The life cycle of creeping avens has also been used by de-Camino-Beck and Lewis (2008) to illustrate their method. The resulting formula for $R_{0}$ is given by Eq. (26) of their paper. Their formula differs from Eq. (13) in several ways and we now briefly discuss these differences. First, note that 
Eq. (26) in de-Camino-Beck and Lewis (2008) contains a small error. For the formula to be correct the parameter $\tau_{45}$ introduced by de-Camino-Beck and Lewis has to be defined as $\tau_{45}=s_{45} s_{54} /\left(1-s_{44}\right)+s_{55}$ instead of $\tau_{45}=$ $s_{45} s_{54} /\left(\left(1-s_{44}\right)\left(1-s_{55}\right)\right)$. With this correction, Eq. (26) in de-Camino-Beck and Lewis (2008) can be re-written as

$$
\begin{aligned}
R_{0}= & f_{33}+\frac{f_{35} s_{54} s_{43}}{\left(1-s_{44}\right)\left(1-s_{55}\right)-s_{45} s_{54}}+\frac{f_{13} s_{32} s_{21}}{1-s_{22}} \\
& +\frac{f_{15} s_{54} s_{43} s_{32} s_{21}}{\left(1-s_{22}\right)\left(\left(1-s_{44}\right)\left(1-s_{55}\right)-s_{45} s_{54}\right)} \\
& +\frac{f_{34} s_{43}}{1-s_{44}}+\frac{f_{34} s_{43} s_{45} s_{54}}{\left(1-s_{44}\right)\left(\left(1-s_{44}\right)\left(1-s_{55}\right)-s_{45} s_{54}\right)} \\
& +\frac{f_{14} s_{43} s_{32} s_{21}}{\left(1-s_{44}\right)\left(1-s_{55}\right)}+\frac{f_{14} s_{43} s_{32} s_{21} s_{45} s_{54}}{\left(1-s_{22}\right)\left(1-s_{44}\right)\left(\left(1-s_{22}\right)\left(1-s_{55}\right)-s_{45} s_{54}\right)}
\end{aligned}
$$

Surprisingly, the formula derived by de-Camino-Beck and Lewis contains eight terms while Eq. (13) contains only six terms. The first four terms on the righthand side of Eq. (14) occur in identical form in Eq. (13). The two terms in the third row on the right-hand side of Eq. (14) both contain the fecundity loop $f_{34} s_{43}$ while the two terms in the fourth row both contain the fecundity loop $f_{14} s_{43} s_{32} s_{21}$. After some algebraic manipulation it can be shown that the terms in the third and fourth row of the right-hand side of Eq. (14) equal the second and fifth term on the right-hand side of Eq. (13), respectively. Thus, in contrast to our algebraic formula it appears that the graph-reduction method does not necessarily lead directly to a formula for $R_{0}$ where each summand describes a separate fertility loop as claimed by de-Camino-Beck and Lewis (2007, 2008).

As a second example we consider a population with both age- and spatial structure. At census individuals are classified as being either in their first or second year and as living in one of two possible habitats. Individuals in the second year reproduce within their habitat and then die and juveniles disperse after their first birthday. The corresponding life cycle is shown in Fig. 2(a).

According to Eq. (4) the characteristic equation of the corresponding population projection matrix $\mathrm{A}$ can be written as

$$
1=f_{13} s_{31} z^{-1}+f_{24} s_{42} z^{-1}+f_{13} s_{32} f_{24} s_{41} z^{-2}-f_{13} s_{31} f_{24} s_{42} z^{-2} .
$$

The right-hand side of Eq. (15) shows two summands containing the factor $z^{-2}$. The first of these summands corresponds to a loop containing two fertility parameters wile the second of these summands corresponds to the product of two unconnected fertility loops. Thus, Eq. (15) corresponds to a polynomial equation of degree two and therefore $R_{0}$ cannot be expressed in a simple manner.

The situation changes if dispersal of one-year old individuals is random, i.e., if juveniles from each habitat have the same probability to have their reproductive phase in habitat 1 or 2 . In this case all juveniles enter a dispersal (a)

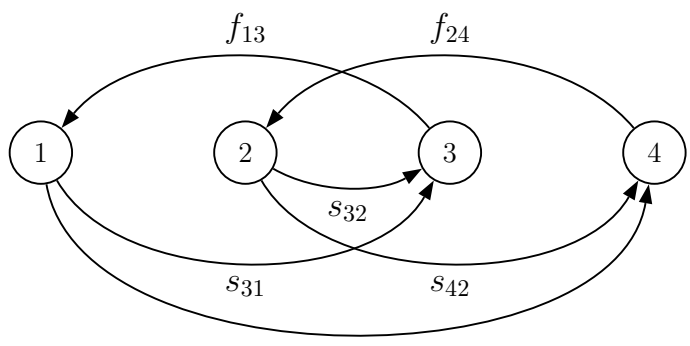

$s_{41}$

(b)

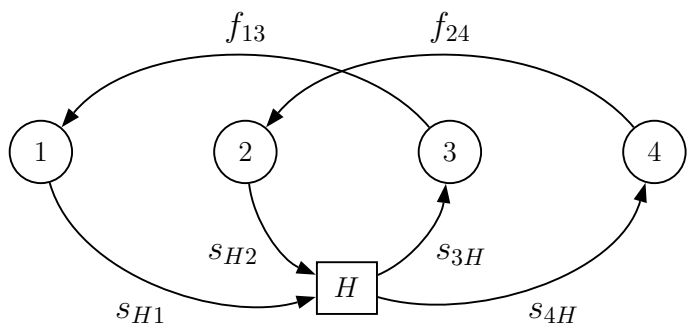

Fig. 2 Hypothetical life cycle of an organism occupying two habitats. Individuals are classified as juveniles (in their first year) or as adults (in their second year) and as living in habitat one or two. Juveniles in habitat 1 and 2 are assigned i-state (1) and (2), respectively. Adults in habitat 1 and 2 are assigned i-state (3) and (4), respectively. Adults reproduce once within their habitat and then die. Juveniles disperse before becoming sexually mature. Dispersal is either restricted (a) or global (b). In the latter case, the global dispersal pool $H$ that is entered by all maturing individuals and from whic hey are redistributed.

pool at some time point $\Delta$ between two censuses and the dispersal pool becomes a hidden i-state in the sense introduced in the previous section. Fig. 2(b) shows the life-cycle of the same organism but with the hidden i-state shown explicitly. Rewriting Eq. (15) for this new life cycle gives

$$
1=f_{13} s_{3 H} s_{H 1} z^{-1}+f_{24} s_{4 H} s_{H 2} z^{-1} .
$$

In particular, no loop with more than one fertility parameter exists and the previously unconnected fertility loops are now connected. As a consequence, $z^{-1}$ occurs linearly in Eq. (16) and we have $R_{0}=f_{13} s_{3 H} s_{H 1}+f_{24} s_{4 H} s_{H 2}$. Note that this example fits the pattern described by Eq. (11) with $\mathrm{S}_{11}=0=\mathrm{S}_{22}$ and

$$
\mathrm{S}_{21}=\left(\begin{array}{ll}
s_{3 H} s_{H 1} & s_{3 H} s_{H 2} \\
s_{4 H} s_{H 1} & s_{4 H} s_{H 2}
\end{array}\right) \text { and } \mathrm{F}_{12}=\left(\begin{array}{cc}
f_{13} & 0 \\
0 & f_{24}
\end{array}\right)
$$




\section{Discussion}

The basic reproduction ratio $R_{0}$ as calculated from a population projection matrix A determines whether a population is growing or shrinking in the long run. The same information can be extracted from the dominant eigenvalue $\lambda_{\mathrm{d}}$ of $\mathrm{A}$. An advantage of $R_{0}$ over $\lambda_{\mathrm{d}}$ is that the former quantity can sometimes be calculated analytically in models where the latter quantity can only be calculated numerically. Recently, de-Camino-Beck and Lewis $(2007,2008)$ presented a graph-reduction method that for certain life cycles leads to an expression for $R_{0}$ directly from a life cycle graph. The advantage of their method is that the resulting expression for $R_{0}$ is the expected number of offspring per fertility loop summed over all fertility loops. An expression for $R_{0}$ having this form can be a useful complement to elasticity and sensitivity analysis (Caswell, 2001) in developing strategies for pest control and conservation. In particular, fertility loops can be identified that on their own suffice to maintain population growth.

From the work of de-Camino-Beck and Lewis $(2007,2008)$ it is clear that $R_{0}$ can not always be written in this mathematically pleasing and biologically informative manner. However, the conditions when this is possible, and therefore, when the approach taken by these authors is useful in the first place, were not specified. Mathematically speaking, this is the case if and only if the next-generation matrix $G$ has only a single non-zero eigenvalue. Here we prove that this condition can be expressed in several mathematically equivalent but biologically more insightful manners.

In the first part of this article we present a fully algebraic counterpart to the graph-reduction method of de-Camino-Beck and Lewis $(2007,2008)$. In particular, the expressions for $R_{0}$ as given by Eq. (6) and (8) have the same property as the expression one obtains from the graph-reduction method: each summand represents the expected number of offspring for a given fertility loop. We then show that in terms of loops in the life cycle graph the matrix $G$ has a single non-zero eigenvalue if (i) all fertility loops are pairwise connected and (ii) all fertility loops contain only a single fertility parameter. Given (i), we show that (ii) if and only if an i-state exists that is passed by all fertility loops. For the usual case of irreducible life cycles the condition becomes that all paths from birth to reproduction should pass through a common i-state. Importantly, these requirements do not imply the existence of a single birth state. Thus, even in life cycles with more than one birth state it can be possible to calculate $R_{0}$ according to Eq. (6) or (8).

The above conditions are sufficient but generally not necessary for $G$ to have a single non-zero eigenvalue. However, if life history states are taken into account that are not visible at population census but only present between censuses and if such a life history state exists that is passed by all fertility loops then the above conditions are sufficient and generically also necessary. Thus, the next-generation matrix has a single non-zero eigenvalue if and generically only if all fertility loops contain a single fertility parameter and an i-state exists, overt or hidden, that is passed by all fertility loops. This result allows for a simple and biologically meaningful interpretation of $R_{0}$. Whenever $R_{0}$ can be calculated according to Eq. (6) or (8) then $R_{0}$ gives the expected number of new individuals that enter the common i-state deriving from a single individual in this i-state.

An example of the explicit introduction of such hidden i-states to arrive at an algebraically simple model can be found in Metz et al. (2009). These authors show that the outcome of an evolutionary invasion analysis of a life history problem in which the life cycle can be partitioned into a phase affected by the resident community through an environmental feedback loop and a phase in which life history transitions are affected by mutations can be predicted from an optimization procedure given the transition between the two phases can be described by a matrix of rank one. This idea is treated from a more general perspective in Section 3.3 in Rueffler et al. (2012).

We conclude by noting that if fertility loops with more than one fertility parameter exist or no i-state, overt or hidden, exists that is passed by all fertility loops, then no additional biological insight can be expected from the graph-reduction method or its algebraic counterpart. Life cycles with multiple modes of reproduction, as in the example of cat's ear (Hypochaeris radicata) discussed in de-Camino-Beck and Lewis (2008), or with spatial structure, as in the example shown in Fig. 2(a), commonly show these features. In these cases, $R_{0}$ can frequently only be calculated numerically. However, even then all hope on analytical results is not lost, as for some purposes, in particular the calculation of ESSes, one can sometimes fall back on less easily interpretable, but algebraically equally simple loop-based expressions. Define for A a quantity $Q_{\mathrm{A}}:=-\operatorname{det}(\mathrm{I}-\mathrm{A})$ and define $Q_{\mathrm{G}}$ and $Q_{\mathrm{S}}$ similarly for $\mathrm{G}$ and $\mathrm{S}$, respectively. For purely age-dependent models $Q_{\mathrm{A}}=R_{0}-1$ (Appendix F). This generalizes to the case where all fertility loops pass through a common i-state in that $Q_{\mathrm{A}}=\left(-Q_{\mathrm{S}}\right)\left(R_{0}-1\right)$ (Appendix $\mathrm{F}$ ), and hence is sign equivalent to $R_{0}-1$ (since by the next result $\left.Q_{\mathrm{S}}<0\right)$. In general, i.e., also for cases in which $\mathrm{G}$ has more than a single non-zero eigenvalue, if $Q_{\mathrm{A}}>0$ or $Q_{\mathrm{G}}>0$ also $R_{0}>1$ (Metz and Leimar, 2011). To get a converse statement we have to consider a parametric model family, say parametrized with a vector $\boldsymbol{x} \in \mathcal{X}$, with the components of the matrices $\mathrm{S}$ and $\mathrm{F}$ continuous in $\boldsymbol{x}$, so that we may write $\mathrm{A}(\boldsymbol{x}), \mathrm{G}(\boldsymbol{x}), Q_{\mathrm{A}}(\boldsymbol{x})$ and $Q_{\mathrm{G}}(\boldsymbol{x})$. Then, if we know some value of $\boldsymbol{x}$, say $\boldsymbol{x}^{*}$, such that $R_{0}\left(\boldsymbol{x}^{*}\right)=1$ then $R_{0}(\boldsymbol{x})<1$ for all $\boldsymbol{x}$ that connect to $\boldsymbol{x}^{*}$ by a continuous path along which $Q_{\mathrm{A}}(\boldsymbol{x})$ or $Q_{\mathrm{G}}(\boldsymbol{x})<0$ (Metz and Leimar, 2011). Such situations occur for example in evolutionary ecology, where a resident type has $R_{0}=1$ in the environment that it sets itself. Hence, when $\mathcal{X}$ is path connected a strategy $\boldsymbol{x}^{*}$ is an ESS if and only if in the environment set by it $Q_{\mathrm{A}}(\boldsymbol{x})<0$ or $Q_{\mathrm{G}}(\boldsymbol{x})<0$ for any $\boldsymbol{x} \neq \boldsymbol{x}^{*} . Q_{\mathrm{A}}$ has the computational advantage of being affine in the components of $\mathrm{A}$. This affineness is not only computationally advantageous, it also makes for the best possible interpretability of any results based on its use as a fitness proxy. Moreover, up to multiplication with a positive constant it is the only quantity with this property that is sion equivalent to $R_{0}-1$ locally around $R_{0}-1=0$ (Appendix F). Rueffler et al. (2012) demonstrate how $Q_{\mathrm{A}}$ 
can be used to derive qualitative results about how life cycle structures affect evolution.

Acknowledgements C.R. received funding from the Vienna Science and Technology Fund (WWTF) through project MA07-015. H.M. benefited from the support of the Chair Modélisation Mathématique et Biodiversité VEOLIA-École Polytechnique-MNHN-F X.

\section{References}

Caswell, H. 2001. Matrix Population Models. 2nd ed. Sinauer.

Cushing, J., and Z. Yicang. 1994. The net reproductive value and stability in matrix population models. Natural Resources Modeling 8:297-333.

de-Camino-Beck, T., and M. A. Lewis. 2007. A new method for calculating net reproductive rate from graph reduction with applications to the control of invasive species. Bulletin of Mathematical Biology 69:1341-1354.

2008. On net reproductive rate and the timing of reproductive output.

The American Naturalist 172:128-139.

Diekmann, O., J. A. P. Heesterbeek, and J. A. J. Metz. 1990. On the definition and the computation of the basic reproduction ratio $R_{0}$ in models for infectious diseases in heterogeneous populations. Journal of Mathematical Biology 28:365-382.

Horn, R., and C. Johnson. 1985. Matrix Analysis. Cambridge University Press.

Hurford, A., D. Cownden, and T. Day. 2010. Nex-generation tools for evolutionary analysis. Journal of the Royal Society Interface 7:561-571.

Li, C.-K., and H. Schneider. 2002. Applications of Perron-Frobenius theory to population dynamics. Journal of Mathematical Biology 44:450-462.

Mason, S. 1956. Feedback theory - Further properties of signal flow graphs. Proceedings of IRE 44:920-926.

Metz, J. A. J. submitted. On the concept of individual in ecology and evolution. Journal of Mathematical Biology

Metz, J. A. J., and O. Diekmann. 1986. The Dynamics of Physiologically Structured Populations, vol. 68 of Lecture Notes in Biomathematics. Springer Verlag, Berlin, Germany.

Metz, J. A. J., P. G. L. Klinkhamer, and T. J. de Jong. 2009. A different model to explain delayed germination. Evolutionary Ecology Research 11:177-190. Metz, J. A. J., and O. Leimar. 2011. A simple fitness proxy for ess calculation in structured populations with continuous traits, with applications to the evolution of haplo-diploids and genetic dimorphisms. Journal of Biological Dynamics 5:163-190.

Rueffler, C., J. A. J. Metz, and T. J. M. Van Dooren. 2012. What life cycle graphs can tell about the evolution of life histories. Journal of Mathematical Biology. DOI 10.1007/s00285-012-0509-x.

Weppler, T., P. Stoll, and J. Stöcklin. 2006. The relative importance of sexual and clonal reproduction for population growth in the long-lived alpine plant Geum reptans. Journal of Ecology 94:869-879. 


\section{A Derivation of Eq. (4) - (6)}

The basic ideas of loop formulas go back to Mason (1956). Often even formulas similar to ours are attributed to this paper (e.g. Caswell, 2001, p. 181). However, Mason only describes the idea of loop based calculation procedures in an electrical engineering context without giving results that are directly applicable to the population dynamical situation. The formula in Caswell (2001) also do not cover our specific case. Hence, we below give a full derivation of Eq. (4). Thus, we derive an expression for the characteristic equation of the next-generation matrix

$$
0=\operatorname{det}\left(z \mathbf{I}-\mathbf{F}(\mathbf{I}-\mathrm{S})^{-1}\right)
$$

in terms of loops in the life cycle. The largest number $z$ satisfying Eq. (A1) is known as the basic reproduction ratio $R_{0}$. Multiplying both sides with $\operatorname{det}(\mathrm{I}-\mathrm{S})$ and using that for any two $n \times n$ matrices $\mathrm{A}$ and $\mathrm{B}$ holds $\operatorname{det} \mathrm{A} \operatorname{det} \mathrm{B}=\operatorname{det}(\mathrm{AB})$ we get

$$
0=\operatorname{det}(z(\mathbf{I}-\mathrm{S})-\mathbf{F})=\operatorname{det}(z \mathbf{I}-(z \mathbf{S}+\mathbf{F})) .
$$

We continue by considering the case of an arbitrary nonnegative $n \times n$-matrix M. Let $\alpha \subseteq\{1, \ldots, n\}$ be an index set and let $\mathcal{P}(\alpha)$ denote the power set of $\alpha$ and $\mathcal{P}_{m}(\alpha)$ the set of all subsets of $\alpha$ containing exactly $m$ elements. With $\mathrm{M}_{\alpha}$ we denote the sub-matrix of $\mathrm{M}$ with indices in $\alpha$. Then

$$
\begin{aligned}
\operatorname{det}(z \mathbf{I}-\mathrm{M})= & z^{n}-\sum_{\alpha \in \mathcal{P}_{1}} \operatorname{det} \mathrm{M}_{\alpha} z^{n-1}+\sum_{\alpha \in \mathcal{P}_{2}} \operatorname{det} \mathrm{M}_{\alpha} z^{n-2}-\ldots \\
& +(-1)^{n-1} \sum_{\alpha \in \mathcal{P}_{n-1}} \operatorname{det} \mathrm{M}_{\alpha} z+(-1)^{n} \operatorname{det} \mathrm{M}
\end{aligned}
$$

(e.g. Horn and Johnson 1985 , p. 42 ), where $\mathcal{P}_{k}$ stands for $\mathcal{P}_{k}(\{1, \ldots, n\})$. By multiplying both sides with $z^{-n}$ it is clear the characteristic equation can be written as

$$
\begin{aligned}
1= & \sum_{\alpha \in \mathcal{P}_{1}} \operatorname{det} \mathrm{M}_{\alpha} z^{-1}-\sum_{\alpha \in \mathcal{P}_{2}} \operatorname{det} \mathrm{M}_{\alpha} z^{-2}+\ldots \\
& +(-1)^{n-2} \sum_{\alpha \in \mathcal{P}_{n-1}} \operatorname{det} \mathrm{M}_{\alpha} z^{-(n-1)}+(-1)^{n-1} \operatorname{det} \mathrm{M}^{-n} .
\end{aligned}
$$

Starting point of our further considerations is the Leibniz formula of the determinan of a $n \times n$ matrix $\mathrm{M}, \operatorname{det} \mathrm{M}=\sum_{\sigma \in S_{n}} \operatorname{sgn}[\sigma] \prod_{i=1}^{n} m_{i \sigma(i)}$, where $\sigma$ is a permutation of the set $\{1, \ldots, n\}$ and $S_{n}$ the set of all permutations. The sign of a permutation is defined as $\operatorname{sgn}[\sigma]=(-1)^{N(\sigma)}$, where $N(\sigma)$ is number of inversions in $\sigma$.

Recall from elementary group theory that any finite permutation can be decomposed into disjoint permutation cycles where a permutation cycle of length $k$ is defined as a permutation $\sigma$ of a set $\left\{a_{1}, \ldots, a_{k}\right\}$ such that $\sigma\left(a_{i}\right)=a_{i+1}$ and $\sigma\left(a_{k}\right)=a_{1}$. The sign of a permutation cycle is given by the number of inversions. Thus, for a permutation cycle of length $k$ we have $\operatorname{sgn}[\sigma]=k-1$. Let $c_{1}, \ldots, c_{j}$ denote the permutation cycles of a permutation $\sigma$ of length $n$ in cycle notation, $\sigma=c_{1} \ldots c_{j}, d(\sigma)$ the number of disoint permutation cycles in a permutation $\sigma$ and $\left|c_{i}\right|$ the length of a permutation cycle. Then $\operatorname{sgn}[\sigma]=\operatorname{sgn}\left[c_{1}\right] \ldots \operatorname{sgn}\left[c_{j}\right]=(-1)^{\left(\left(\left|c_{1}\right|-1\right)+\ldots+\left(\left|c_{j}\right|-1\right)\right)}=(-1)^{\left(\left|c_{1}\right|+\ldots+\left|c_{j}\right|-j\right)}=$ $(-1)^{n-j}=(-1)^{n-d(\sigma)}$. Thus, $\operatorname{sign}[\sigma]=(-1)^{n-d(\sigma)}$.

The set of permutations $S_{|\alpha|}$ can be decomposed into a partition with elements $C_{j}:=$ $\left\{\sigma \in S_{|\alpha|}: d(\sigma)=j\right\}$ and $j \in\{1, \ldots,|\alpha|\}$. Thus, for each $i$ the set $C_{i}$ contains the permutations that consist of exactly $j$ permutation cycles. Hence, the determinant can be rewritten as

$$
\begin{aligned}
\operatorname{det} \mathrm{M} & =\sum_{j=1}^{n} \sum_{\sigma \in C_{j}}(-1)^{n-j} \prod_{i=1}^{n} m_{i \sigma(i)} \\
& =\sum_{\sigma \in C_{1}}(-1)^{n-1} \prod_{i=1}^{n} m_{i \sigma(i)}+\sum_{\sigma \in C_{2}}(-1)^{n-2} \prod_{i=1}^{n} m_{i \sigma(i)}+\ldots+\sum_{\sigma \in C_{n}} \prod_{i=1}^{n} m_{i \sigma(i)}
\end{aligned}
$$

Let $l \in\{1, \ldots, n\}$ and $c=\left(l \sigma(l) \sigma^{2}(l) \ldots \sigma^{|c|-1}(l)\right)$ be a permutation cycle in cycle notation. Then $L=m_{l \sigma|c|-1(l)} m_{\sigma^{|c|-1}(l) \sigma^{|c|-2}(l)} \ldots m_{\sigma(l) l}$ is a loop of length $|\breve{L}|=|c|$. Thus, a permutation $\sigma \in C_{j}$ corresponds to the product of $j$ pairwise unconnected loops Lhus, a permutation $\sigma \in L_{j}$. From this follows that we can rearrange Eq. (A3) in the following way:

$$
\begin{aligned}
& 1=\sum_{\alpha \in \mathcal{P}_{1}} \operatorname{det} \mathrm{M}_{\alpha} z^{-1}-\sum_{\alpha \in \mathcal{P}_{2}} \operatorname{det} \mathrm{M}_{\alpha} z^{-2}+\ldots+(-1)^{n-2} \sum_{\alpha \in \mathcal{P}_{n-1}} \operatorname{det} \mathrm{M}_{\alpha} z^{-(n-1)} \\
& =\sum_{\left\{L \in \mathcal{L}_{\mathrm{M}}:|\breve{L}|=1\right\}} \hat{L}
\end{aligned}
$$

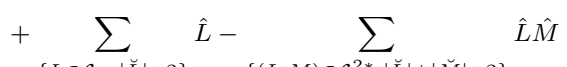

$$
\begin{aligned}
& +\sum_{\left\{L \in \mathcal{L}_{\mathrm{M}}:|\breve{L}|=3\right\}} \hat{L}-\sum_{\left\{(L, M) \in \mathcal{L}_{\mathrm{M}}^{2 *}:|\breve{L}|+|\breve{M}|=3\right\}} \hat{L} \hat{M}+\sum_{\left\{(L, M, N) \in \mathcal{L}_{\mathrm{M}}^{3 *}:|\breve{L}|+|\breve{M}|+|\breve{N}|=3\right\}} \hat{L} \hat{M} \hat{N} \\
& +\sum_{\left\{L \in \mathcal{L}_{\mathrm{M}}:|\breve{L}|=n\right\}} \hat{L}-\sum_{\left\{(L, M) \in \mathcal{L}_{\mathrm{M}}^{2 *}:|\breve{L}|+|\breve{M}|=n\right\}} \hat{L} \hat{M}+\ldots \\
& +(-1)^{n-1} \sum_{\left\{\left(L_{i}, \ldots, L_{n}\right) \in \mathcal{L}_{\mathrm{M}}^{n *}: \sum_{i=1}^{n}\left|\breve{L}_{i}\right|=n\right\}} \prod_{i=1}^{n} \hat{L}_{i} \\
& =\sum_{\mathcal{L}_{\mathrm{M}}} L z^{-|\breve{L}|}+\sum_{\mathcal{L}_{\mathrm{M}}^{2 *}} L M z^{-(|\breve{L}|+|\breve{M}|)}-\sum_{\mathcal{L}_{\mathrm{M}}^{3 *}} L M N z^{-(|\breve{L}|+|\breve{M}|+|\breve{N}|)}+\ldots, \\
& +(-1)^{n-1} \operatorname{det} \mathrm{M} z^{-n}
\end{aligned}
$$

where $\hat{L}:=L z^{-|\breve{L}|}$ and $\mathcal{L}_{\mathrm{M}}^{k *}$ denotes the $k$-fold Cartesian product over the set of loops $\mathcal{L}_{\mathrm{M}}$. The star indicates that $k$-tuples in which not all loops are unconnected to each other are excluded from the Cartesian product.

By replacing $\mathrm{M}$ with $z \mathrm{~S}+\mathrm{F}$ Eq. (A7) can be rewritten as

$$
\begin{aligned}
0 & =1-\sum_{\mathcal{L}_{\mathrm{A}}} L \frac{z^{|L|_{\mathrm{s}}}}{z^{|L|}}+\sum_{\mathcal{L}_{\mathrm{A}}^{2 *}} L M \frac{z^{|L|_{\mathrm{s}}+|M|_{\mathrm{s}}}}{z^{|\breve{L}|+|M|}}-\sum_{\mathcal{L}_{\mathrm{A}}^{3 *}} L M N \frac{z^{|L|_{\mathrm{s}}+|M|_{\mathrm{s}}+|N|_{\mathrm{s}}}}{z^{|\breve{L}|+|\breve{M}|+|\breve{N}|}+\ldots}{ }=1-\sum_{\mathcal{L}_{\mathrm{A}}} L z^{-|L|_{\mathrm{f}}}+\sum_{\mathcal{L}_{\mathrm{A}}^{2 *}} L M z^{-\left(|L|_{\mathrm{f}}+|M|_{\mathrm{f}}\right)}-\sum_{\mathcal{L}_{\mathrm{A}}^{3 *}} L M N z^{-\left(|L|_{\mathrm{f}}+|M|_{\mathrm{f}}+|N|_{\mathrm{f}}\right)}+\ldots, \quad(\text { A } 8)
\end{aligned}
$$

where the expressions $|L|_{\mathrm{f}}$ and $|L|_{\mathrm{s}}$ denote the number of fertility parameters $f_{l k}$ and the number of transition parameters $s_{l k}$ in loop $L$, respectively. Furthermore, $|\breve{L}|$ indicates the number of i-states traversed by loop $L$ and therefore also the number of demographic parameters in $L$. Thus, $|\breve{L}|=|L|_{\mathrm{f}}+|L|_{\mathrm{s}}$. 
By sorting the terms into those containing fertility parameters and those that do not, Eq. (A8) can be rearranged in the following way:

$$
\begin{aligned}
& 0=-\sum_{\mathcal{L}_{\mathrm{f}, \mathrm{A}}} L z^{-|L|_{\mathrm{f}}}+\sum_{\mathcal{L}_{\mathrm{f}}{ }^{*} \times \mathcal{L}_{\mathrm{A}}} L M z^{-\left(|L|_{\mathrm{f}}+|M|_{\mathrm{f}}\right)}-\sum_{\mathcal{L}_{\mathrm{f},}^{*} \times \mathcal{L}_{\mathrm{A}}^{*} \times \mathcal{L}_{\mathrm{A}}} L M N z^{-\left(|L|_{\mathrm{f}}+|M|_{\mathrm{f}}+|N|_{\mathrm{f}}\right)}+\ldots \\
& +1-\sum_{\mathcal{L}_{\mathrm{s}}} L+\sum_{\mathcal{L}^{2 *}} L M-\sum_{\mathcal{L}^{3 *}} L M N+\ldots \\
& =-\sum_{\mathcal{L}_{\mathrm{f}, \mathrm{A}}} L z^{-|L|_{\mathrm{f}}}+\sum_{\mathcal{L}^{*}} L M z^{-\left(|L|_{\mathrm{f}}+|M|_{\mathrm{f}}\right)} \\
& -\sum_{\mathcal{L}_{\mathrm{f}, \mathrm{A}}{ }^{*} \mathcal{L}_{\mathrm{A}} * \mathcal{L}_{\mathrm{A}}^{*}} L M N z^{-\left(|L|_{\mathrm{f}}+|M|_{\mathrm{f}}+|N|_{\mathrm{f}}\right)}+\ldots+\operatorname{det}(\mathrm{I}-\mathrm{S}) .
\end{aligned}
$$

The last simplification follows from Eq. (A7) by choosing $\mathrm{M}=\mathrm{S}$ and $z=1$. Dividing both sides by $-\operatorname{det}(\mathbf{I}-\mathrm{S})$ results in Eq. (4) in the main text.

Eq-(5) in the main text can be simplified. Recall the notation introduced after Eq. (6). With this notation at hand and by using Eq. (A7) the numerator of Eq. (5) can be written

$$
\sum_{\mathcal{L}_{\mathrm{f}, \mathrm{A}}} L\left(1-\sum_{\mathcal{L}_{\backslash} \backslash \breve{L}} M+\sum_{\mathcal{L}_{S_{\backslash}^{2 *}}^{2 *}} M N-\ldots\right)=\sum_{\mathcal{L}_{\mathrm{f}, \mathrm{A}}} L \operatorname{det}\left(\mathrm{I}-\mathrm{S}_{\backslash \breve{L}}\right),
$$

resulting in Eq. (6) in the main text.

\section{B Proof of Lemma 2}

In this appendix we prove that if all fertility loops contain only a single fertility parameter then all fertility loops are mutually connected if and only if an i-state exists that is shared by all fertility loops. One implication is trivial. If all such loops share a common i-state, then they are mutually connected. The remainder of this appendix deals with the reverse implication.

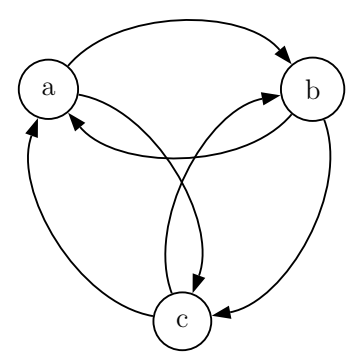

Fig. B1 Life cycle containing the three mutually connected loops $L_{1}=P_{a b} P_{b a}, L_{2}=$ $P_{b c} P_{c b}$ and $L_{3}=P_{c a} P_{a c}$. For these tree loops no shared i-state exists. This life cycle contains two more loops: $L_{4}=P_{a b} P_{b c} P_{c a}$ and $L_{5}=P_{a c} P_{c b} P_{b a}$

For life cycles with one or two fertility loops the statement is trivial. For life cycles with more than two fertility loops we prove the statement by induction. Assume that three mutually connected fertility loops $L_{1}, L_{2}$ and $L_{3}$ exist. If one of them is a self loop the statement is obviously true. Assume none of them is a self loop. We then prove the statement by contradiction. Thus, assume $\left\{L_{1}\right\} \cap\left\{L_{2}\right\} \cap\left\{L_{3}\right\}=\emptyset$. Let $a, b, c$ denote i-states in the life cycle such that $a \in\left\{L_{1}\right\} \cap\left\{L_{3}\right\}, b \in\left\{L_{1}\right\} \cap\left\{L_{2}\right\}$ and $c \in\left\{L_{2}\right\} \cap\left\{L_{3}\right\}$ with $a \neq b$, $a \neq c$ and $b \neq c$. A path is defined as a sequence of demographic parameters $s_{l k}, f_{l k}$ that lead from one i-state to another i-state without passing through any i-state more than once. The path transmission $P_{v u}$ equals the product of the demographic parameters along the path leading from $u$ to $v$. Here we use the terms path and path transmission synonymously and often denote a path with its transmission. Loops in a life cycle can be written as a concatenation of paths, e.g., $L=P_{u v} P_{v u}$ or $M=P_{w v} P_{v u} P_{u w}$. Thus, the life cycle under consideration has the structure shown in Fig. B1 where the arrows correspond to paths and not necessarily to single demographic parameters as in Fig. 1 in the main part. Thus, next to the loops $L_{1}=P_{a b} P_{b a}, L_{2}=P_{b c} P_{c b}$ and $L_{3}=P_{a c} P_{c a}$ two more loops exist. These are $L_{4}:=P_{a b} P_{b c} P_{c a}$ and $L_{5}:=P_{a c} P_{c b} P_{b a}$. Each path $P_{a b}, P_{b a}, P_{b c}, P_{c b}, P_{a c}, P_{c a}$ contains either exactly one fertility parameter $f_{l k}$ or no fertility parameter. We indicate the presence of a fertility parameter in a path with the subscript f, e.g. $P_{a b, \mathrm{f}}$. Then the three fertility parameters can be distributed over the six different paths in $2^{3}$ different ways with resulting loops $L_{4}$ and $L_{5}$ as follows:

$$
\begin{aligned}
& \left\{P_{b a, \mathrm{f}}, P_{c b, \mathrm{f}}, P_{a c, \mathrm{f}}\right\} \Longleftrightarrow L_{4}=P_{b a, \mathrm{f}} P_{c b, \mathrm{f}} P_{a c, \mathrm{f}} \wedge L_{5}=P_{c a} P_{b c} P_{a b} \\
& \left\{P_{b a, \mathrm{f}}, P_{c b, \mathrm{f}}, P_{c a, \mathrm{f}}\right\} \Longleftrightarrow L_{4}=P_{b a, \mathrm{f}} P_{c b, \mathrm{f}} P_{a c} \wedge L_{5}=P_{c a, \mathrm{f}} P_{b c} P_{a b} \\
& \left\{P_{b a, \mathrm{f}}, P_{b c, \mathrm{f}}, P_{a c, \mathrm{f}}\right\} \Longleftrightarrow L_{4}=P_{b a, \mathrm{f}} P_{c b} P_{a c, \mathrm{f}} \wedge L_{5}=P_{c a} P_{b c, \mathrm{f}} P_{a b} \\
& \left\{P_{a b, \mathrm{f}}, P_{c b, \mathrm{f}}, P_{a c, \mathrm{f}}\right\} \Longleftrightarrow L_{4}=P_{b a} P_{c b, \mathrm{f}} P_{a c, \mathrm{f}} \wedge L_{5}=P_{c a} P_{b c} P_{a b, \mathrm{f}} \\
& \left\{P_{a b, \mathrm{f}}, P_{b c, \mathrm{f}}, P_{a c, \mathrm{f}}\right\} \Longleftrightarrow L_{4}=P_{b a} P_{c b} P_{a c, \mathrm{f}} \wedge L_{5}=P_{c a} P_{b c, \mathrm{f}} P_{a b, \mathrm{f}} \\
& \left\{P_{b a, \mathrm{f}}, P_{b c, \mathrm{f}}, P_{c a, \mathrm{f}}\right\} \Longleftrightarrow L_{4}=P_{b a, \mathrm{f}} P_{c b} P_{a c} \wedge L_{5}=P_{c a, \mathrm{f}} P_{b c, \mathrm{f}} P_{a b} \\
& \left\{P_{a b, \mathrm{f}}, P_{c b, \mathrm{f}}, P_{c a, \mathrm{f}}\right\} \Longleftrightarrow L_{4}=P_{b a} P_{c b, \mathrm{f}} P_{a c} \wedge L_{5}=P_{c a, \mathrm{f}} P_{b c} P_{a b, \mathrm{f}} \\
& \left\{P_{a b, \mathrm{f}}, P_{b c, \mathrm{f}}, P_{c a, \mathrm{f}}\right\} \Longleftrightarrow L_{4}=P_{b a} P_{c b} P_{a c} \wedge L_{5}=P_{c a, \mathrm{f}} P_{b c, \mathrm{f}} P_{a b, \mathrm{f}}
\end{aligned}
$$

In each case a loop with more than one fertility parameter exists, contradicting the assumption that no such loops exist. Hence, the loops $L_{1}, L_{2}$ and $L_{3}$ have to have at least on -state in common.

Next consider a life cycle containing the fertility loops $L_{1}, \ldots, L_{n}, L_{n+1}$ which all contain only a single fertility parameter. Assume that the loops $L_{1}, \ldots, L_{n}$ have a least one i-state in common: $\alpha:=\breve{L}_{1} \cap \ldots \cap \breve{L}_{n} \neq \emptyset$. Furthermore, assume that (i) the loop $L_{n+1}$ is connected to all other loops but (ii) not via one of the i-states that is shared by those loops: $\breve{L}_{n+1} \cap \breve{L}_{j} \neq \emptyset$ for all $L_{j} \in\left\{L_{1}, \ldots, L_{n}\right\}$ and $\breve{L}_{n+1} \cap \alpha=\emptyset$. It follows that two loops $L_{l}, L_{k} \in\left\{L_{1}, \ldots, L_{n}\right\}$ exist such that $L_{n+1}$ intersects with $L_{l}$ at an i-state that is not passed by $L_{k}$ and that $L_{n+1}$ intersects with $L_{k}$ at an i-state that is not passed by $L_{l}: \beta:=\left(\breve{L}_{n+1} \cap \breve{L}_{l}\right) \backslash \breve{L}_{k} \neq \emptyset$ and $\gamma:=\left(\breve{L}_{n+1} \cap \breve{L}_{k}\right) \backslash \breve{L}_{l} \neq \emptyset$. If such loops would not exist, then (i) and (ii) could not be fulfilled simultaneously. Let $a, b$ and $c$ denote three i-states such that $a \in \alpha, b \in \beta$ and $c \in \gamma$. Then the life cycle under consideration contains three loops that are connected as shown in Fig. B1. With the above argument follows that then a loop with more than one fertility parameter has to exist, contradicting our assumption that no such loop exists. Thus, $\breve{L}_{n+1} \cap \alpha \neq \emptyset$.

\section{Proof of Theorem 4}

Next we prove Theorem 4. Let A be an irreducible population projection matrix. Let us call a path through which a newborn has to pass till it reaches a state where it can reproduce a maturation path. In particular, in case of a self-loop $f_{k k}$ the maturation path has length zero. Assume that (i) all fertility loops in the life cycle given by A contain exactly one fertility parameter and (ii) an i-state exists that is passed by all fertility loops. Note that for each maturation path a fertility parameter $f_{l k}$ exists that closes the maturation path into a fertility loop. This follows from (i) in combination with the fact that any two i-states are connected by a path (since A is assumed irreducible). Then it follows from (ii) that an i-state 
exists that is passed by all maturation paths. The reverse implication follows immediately since a fertility loop with more than one fertility parameter cannot exist as it would have to pass more than once through that specific i-state.

\section{Proof of Proposition 5}

In this Appendix we prove that if all fertility loops contain only a single fertility parameter and share a common i-state, then $R_{0}$ gives the expected number of offspring surviving until they enter the common i-state that are born over the remaining life time of an individual that has just entered the common i-state. In other words, $R_{0}$ projects the population size in the common i-state from one generation to the next.

Consider a life cycle with $n$ i-states, $m \leq n$ of which are birth states. We number the i-states such that the birth states have indices in $\{1, \ldots, m\}$. Then the rows $m+1$ till $n$ of $\mathrm{G}=\mathrm{F}(\mathrm{I}-\mathrm{S})^{-1}$ contain only zeros:

$$
\mathbf{G}=\mathrm{F}(\mathrm{I}-\mathrm{S})^{-1}=\left(\begin{array}{cccccc}
g_{11} & \cdots & g_{1 m} & g_{1, m+1} & \cdots & g_{1 n} \\
\vdots & \ddots & \vdots & \vdots & \cdots & \vdots \\
g_{m 1} & \cdots & g_{m m} & g_{m, m+1} & \cdots & g_{m n}
\end{array}\right)
$$

Remember that $g_{l k}$ gives the expected number of offspring in birth state $l$ that are born to an individual in i-state $k$.

The eigenvalues of $\mathrm{G}$ are given by the eigenvalues of the $m \times m$ upper left block of $\mathrm{G}$ which we denote by $\mathrm{G}_{11}$. We define $\mathrm{B}=\left[b_{l k}\right]:=(\mathrm{I}-\mathrm{S})^{-1}$ and let $q$ be an i-state that is passed by all fertility loops. Then

$$
g_{l k}=\sum_{j=1}^{n} f_{l j} b_{j k}=\sum_{j=1}^{n} f_{l j} b_{j q} b_{q k}=b_{q k} \sum_{j=1}^{n} f_{l j} b_{j q} .
$$

Thus, all rows of $\mathrm{G}_{11}$ are linear dependent and therefore $\operatorname{rank} \mathrm{G}_{11}=1$. In particular

$$
R_{0}=\sum_{l=1}^{m} g_{l l}=\sum_{l=1}^{m} b_{q l} \sum_{j=1}^{m} f_{l j} b_{j q}
$$

which proves the statement.

\section{E Derivation of Eq. (12)}

Assume that A can be written as in Eq. (11). Then G has the form as given in Eq. (D1). The dominant eigenvalue of $\mathrm{G}, R_{0}$, is given by the dominant eigenvalue of the upper left $m \times m$-matrix on the right-hand side of Eq. (D1), denoted with $\mathrm{G}_{11}$. It is clear that

$$
\mathrm{G}_{11}=\mathrm{F}_{12}\left(\mathrm{I}-\mathrm{S}_{22}\right)^{-1} \mathrm{~S}_{21}\left(\mathrm{I}-\mathrm{S}_{11}\right)^{-1}=\mathrm{F}_{12}\left(\mathrm{I}-\mathrm{S}_{22}\right)^{-1} \boldsymbol{u}_{2} \boldsymbol{u}_{1}^{\mathrm{T}}\left(\mathrm{I}-\mathrm{S}_{11}\right)^{-1}
$$

Let $\boldsymbol{v}$ be the dominant right eigenvector of $\mathrm{G}_{11}$. If we multiply both sides of the eigenvalue equation for $G_{11}$ with $\boldsymbol{u}_{1}^{T}\left(I-S_{11}\right)^{-1}$, we obtain

$\boldsymbol{u}_{1}^{\mathrm{T}}\left(\mathrm{I}-\mathrm{S}_{11}\right)^{-1} \mathrm{G}_{11} \boldsymbol{v}=\boldsymbol{u}_{1}^{\mathrm{T}}\left(\mathrm{I}-\mathrm{S}_{11}\right)^{-1} \mathrm{~F}_{12}\left(\mathrm{I}-\mathrm{S}_{22}\right)^{-1} \boldsymbol{u}_{2} \boldsymbol{u}_{1}^{\mathrm{T}}\left(\mathrm{I}-\mathrm{S}_{11}\right)^{-1} \boldsymbol{v}=\boldsymbol{u}_{1}^{\mathrm{T}}\left(\mathrm{I}-\mathrm{S}_{11}\right)^{-1} \boldsymbol{v} R_{0}$

from which Eq. (12) follows immediately.

\section{F Relationship between $R_{0}, Q_{\mathrm{A}}$ and $Q_{\mathrm{S}}$}

Assume all fertility loops contain only a single fertility parameter and an i-state exists, overt or hidden, that is passed by all fertility loops. Then, with Theorem 8 we know that $R_{0}$ is given by Eq (5). Recall that $Q_{S}:=-\operatorname{det}(I-S)$ and $Q_{\mathrm{A}}:=-\operatorname{det}(\mathrm{I}-\mathrm{A})$. Thus, Eq $(5)$ can be rewritten as

$$
-Q_{\mathrm{S}} R_{0}=\sum_{\mathcal{L}_{\mathrm{f}, \mathrm{A}}} L-\sum_{\mathcal{L}_{\mathrm{f}, \mathrm{A}} \stackrel{*}{\times} \mathcal{L}_{\mathrm{S}}} L M+\sum_{\mathcal{L}_{\mathrm{f}, \mathrm{A}} * \mathcal{L}_{\mathrm{S}} * \mathcal{*}^{\times} \mathcal{L}_{\mathrm{S}}} L M N-\ldots
$$

From the results of Appendix A, in particular Eq. (A9), it is clear that

$$
-\operatorname{det}(\mathrm{I}-\mathrm{A})=\sum_{\mathcal{L}_{\mathrm{f}, \mathrm{A}}} L-\sum_{\mathcal{L}_{\mathrm{f}, \mathrm{A}}{ }^{*} \mathcal{L}_{\mathrm{S}}} L M+\sum_{\mathcal{L}_{\mathrm{f}, \mathrm{A}}{ }^{*} \mathcal{L}_{\mathrm{S}} \stackrel{*}{\times} \mathcal{L}_{\mathrm{S}}} L M N-\ldots-\operatorname{det}(\mathbf{I}-\mathrm{S}) .
$$

Thus, the right-hand side of Eq. (F1) equals $Q_{\mathrm{A}}-Q_{\mathrm{S}}$ so that Eq. (F1) can be rewritten as

$$
Q_{\mathrm{A}}=\left(-Q_{\mathrm{S}}\right)\left(R_{0}-1\right) \text {. }
$$

Note that for purely age-dependent models $-Q_{\mathrm{S}}=1$

That $Q_{\mathrm{A}}$ is affine in the components of $\mathrm{A}$ follows from the fact that this is a property of determinants. Now assume that there exists another function $\mathrm{A} \mapsto Q_{A}^{\prime}$ that is both locally sign equivalent to $R_{0}-1$ and affine in the components of $\mathrm{A}$. In that case $Q_{\mathrm{A}}$ and $Q_{\mathrm{A}}^{\prime}$ have the same sign for $\mathrm{A}$ in an open set of nonnegative matrices containing the set of matrices for which $R_{0}=1$. Consider a matrix such that $R_{0}=1$. Now concentrate on two arbitrary matrix components that are not in the same column or row of $\mathrm{A}$, referred to as $x$ and $y$, while keeping the other matrix components fixed. Then there exist an interval of $x$-values such that there exists a nonlinear function $f$ such that $R_{0}=1$, and hence $Q_{\mathrm{A}}=0=Q_{\mathrm{A}}^{\prime}$, for $y=f(x)$, Then, by the affineness in the matrix components, there exist constants $a, b, c, d, a^{\prime}, b^{\prime}, c^{\prime}$ $d^{\prime}$ such that $Q_{\mathrm{A}}=x f(x) a+x b+f(x) c+d=0$ and $Q_{\mathrm{A}}=x f(x) a^{\prime}+x b^{\prime}+f(x) c^{\prime}+d^{\prime}=0$ Hence $f(x)=(b x+d) /(a x+c)=\left(b^{\prime} x+a^{\prime}\right) /\left(a^{\prime} x+c^{\prime}\right)$. Generically in the remaining matrix components the right hand equality holds good if and only if there exists a constant $k$ (still depending on those other components) such that $a^{\prime}=k a, b^{\prime}=k b, c^{\prime}=k c$ and $d^{\prime}=k d$. (The exceptions occur when be $Q_{A}^{\prime}$ ad $=0$.) For $Q^{\prime}(\mathrm{A})>0$. 